\title{
PRE-TANGO STRUCTURES ON CURVES
}

\author{
Yoshifumi TAKEDA AND KÔJI YoKOGAwA
}

(Received May 29, 2000, revised August 1, 2001)

\begin{abstract}
The pre-Tango structure is a certain invertible sheaf of locally exact differentials on a curve in positive characteristic. On any curve of sufficiently high genus, there necessarily exist pre-Tango structures. Meanwhile, by using the notion of pre-Tango structure, we can construct a form of the affine line over the curve. The completions of the forms are regarded as a generalization of Raynaud's counter-example to the Kodaira vanishing theorem. This suggests that we may have certain pathological phenomena on the completions of all such forms. For the time being, we consider whether every curve of genus greater than one has a pre-Tango structure which brings certain pathological phenomena. In the present article, we give a sufficient condition for the completion of the form which is induced from a pre-Tango structure to have non-closed global differential 1-forms. Moreover, we give a lower bound for the dimension of the locus of the curves which have pre-Tango structures satisfying that sufficient condition, in the moduli space of curves.
\end{abstract}

Introduction. Let $k$ be an algebraically closed field of characteristic $p>0$ and $C$ a smooth projective curve over $k$ of genus $g$. The Tango structure on $C$ is an invertible sheaf $\mathcal{L}$ with $p \cdot \operatorname{deg} \mathcal{L}=2 g-2$ which is contained in the sheaf $\mathcal{B}^{1}$ of the locally exact differentials, in other words, the first sheaf of coboundaries of the de Rham complex of $C$ (see Section 1 for the precise definition). By using Tango structures, Raynaud constructed smooth projective surfaces which give counter-examples to the Kodaira vanishing theorem in positive characteristic, and Russell constructed smooth projective surfaces of general type which have non-trivial global vector fields (cf. Raynaud [6] and Russell [7]). Here we note that their surfaces are completions of forms of the affine line over curves.

However, the condition on the degree for an invertible subsheaf of $\mathcal{B}^{1}$ to be a Tango structure imposes severe restriction on its existence. On the other hand, if there exists an invertible subsheaf of $\mathcal{B}^{1}$ with certain extra conditions, then we can construct smooth projective surfaces, which are completions of forms of the affine line over the curve and which have non-closed global differential 1-forms (cf. Takeda [9]). This suggests that the existence of a certain invertible subsheaf of $\mathcal{B}^{1}$ triggers off certain pathological phenomena in positive characteristic.

In Section 1 of the present article, we introduce the notion of pre-Tango structure, that is a positive invertible subsheaf of $\mathcal{B}^{1}$ (see Definition 1.1), in which the condition on the degree is much more moderate. Indeed, we can show that on any curve of sufficiently high genus, there necessarily exist pre-Tango structures (cf. Corollary 1.5). Moreover, in Section 2, from pre-Tango structures, we obtain forms of the affine line over the curves. Here we are interested

2000 Mathematics Subject Classification. Primary 14H60; Secondary 14F10, 14H10, 14H51. 
in the question whether all of those forms are peculiar to positive characteristic or not. For example, we may pose the question: Are none of the completions of those forms liftable to characteristic zero? Regrettably, the authors do not know what the answer is. The prime cause is that no practical criterion is known.

On the other hand, it is known that every surface which has non-closed global differential 1-forms cannot be lifted over the ring $W_{2}$ of Witt-vectors of length two (cf. Deligne and Illusie [1]). Recall the above-mentioned fact that a certain invertible subsheaf of $\mathcal{B}^{1}$, which is a pre-Tango structure, induces a form of the affine line whose completion has non-closed global differential 1-forms. So, that is not liftable over $W_{2}$. In the latter half of Section 2, we give a sufficient condition for a pre-Tango structure to induce a complete surface having non-closed global differential 1 -forms (cf. Theorem 2.4). Unfortunately, not all pre-Tango structures satisfy this sufficient condition and so the question mentioned above is difficult yet. Meanwhile, there exist pre-Tango structures only on curves of genus greater than one (cf. Theorem 1.4). Under the circumstances, we, for the present, pose the following question:

Does every curve of genus greater than one have a pre-Tango structure which induces a complete surface having non-closed global differential 1-forms?

Still regrettably, the authors do not know the answer to this question. In Section 3, however, we give a lower bound for the dimension of the locus of curves which have pre-Tango structures involving complete surfaces with non-closed global differential 1-forms, in the moduli space (cf. Theorem 3.2).

1. Pre-Tango structures on a curve. Let $C$ be a smooth projective curve of genus $g$ over an algebraically closed field $k$. Suppose that char $k=p>0$. Let $F: \tilde{C} \rightarrow C$ be the relative Frobenius morphism over $k$. Set $\mathcal{B}=F_{*} \mathcal{O}_{\tilde{C}} / \mathcal{O}_{C}$. We then have an exact sequence

$$
0 \rightarrow \mathcal{O}_{C} \rightarrow F_{*} \mathcal{O}_{\tilde{C}} \rightarrow \mathcal{B} \rightarrow 0
$$

where $\mathcal{B}$ is a locally free $\mathcal{O}_{C}$-module of rank $p-1$ and of degree $(p-1)(g-1)$.

Definition 1.1. (1) We call an invertible subsheaf $\mathcal{L}$ of $\mathcal{B}$ a pre-Tango structure on $C$ if $\mathcal{L}$ has positive degree.

(2) If we have, in addition, $p \cdot \operatorname{deg} \mathcal{L}=2 g-2$, then we call the invertible sheaf $\mathcal{L}$ a Tango structure on $C$.

REMARK 1.2. By virtue of the divisibility of the Picard variety of $C$, if we have a pre-Tango structure of degree $n$ on $C$, then we may assume that the pre-Tango structure is of the form $\mathcal{L}^{n}$ with an inclusion into $\mathcal{B}$, where $\mathcal{L}$ is an invertible sheaf of degree one.

The pre-Tango structure has the following notable property:

PROPOSITION 1.3. The existence of a pre-Tango structure on a curve $C$ implies the existence of a stable locally free $\mathcal{O}_{C}$-module $\mathcal{E}$ of rank two whose pull-back $F^{*} \mathcal{E}$ is unstable. 
Proof. Suppose that there exists a pre-Tango structure $\mathcal{L}$ on $C$. By considering an exact sequence

$$
H^{0}\left(C, \mathcal{B} \otimes \mathcal{L}^{-1}\right) \rightarrow H^{1}\left(C, \mathcal{L}^{-1}\right) \rightarrow H^{1}\left(\tilde{C}, F^{*} \mathcal{L}^{-1}\right),
$$

we have an element $\xi$ in $H^{1}\left(C, \mathcal{L}^{-1}\right)$ coming from the section in $H^{0}\left(C, \mathcal{B} \otimes \mathcal{L}^{-1}\right)$ corresponding to the inclusion $\mathcal{L} \hookrightarrow \mathcal{B}$. Moreover, we know that $\xi$ is a non-split extension

$$
0 \rightarrow \mathcal{O}_{C} \rightarrow \mathcal{E} \rightarrow \mathcal{L} \rightarrow 0,
$$

whose Frobenius pull-back $F^{*} \xi$ :

$$
0 \rightarrow \mathcal{O}_{\tilde{C}} \rightarrow F^{*} \mathcal{E} \rightarrow F^{*} \mathcal{L} \rightarrow 0
$$

splits. Here we know that $\mathcal{E}$ is stable but $F^{*} \mathcal{E}$ is unstable. Indeed, we then have a commutative exact diagram

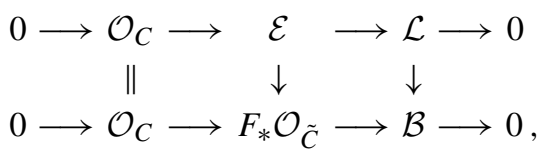

where the last vertical morphism is the inclusion. Therefore, we have that $\mathcal{E}$ is a subsheaf of $F_{*} \mathcal{O}_{\tilde{C}}$. Suppose that $\mathcal{E}$ has a positive invertible subsheaf $\mathcal{M}$. We then have $\mathcal{M} \subset F_{*} \mathcal{O}_{\tilde{C}}$. Taking its adjoint mapping, we have $\mathcal{M}^{p} \subset \mathcal{O}_{C}$. However, this is impossible. So $\mathcal{E}$ is stable. It is clear that $F^{*} \mathcal{E}$ is unstable.

Denote by $F^{*}\left(\mathcal{L}^{-1}\right)$ the mapping $H^{1}\left(C, \mathcal{L}^{-1}\right) \rightarrow H^{1}\left(\tilde{C}, F^{*} \mathcal{L}^{-1}\right)$ for an invertible sheaf $\mathcal{L}$. Tango ([10]) introduced an invariant $\boldsymbol{n}(C)$ concerning the injectivity of $F^{*}\left(\mathcal{L}^{-1}\right)$. Namely, $\boldsymbol{n}(C)$ is the maximal degree of invertible subsheaves of $\mathcal{B}$. Since the kernel of the map $F^{*}\left(\mathcal{L}^{-1}\right)$ is $H^{0}\left(C, \mathcal{B} \otimes \mathcal{L}^{-1}\right)=\operatorname{Hom}(\mathcal{L}, \mathcal{B})$, the positivity of $\boldsymbol{n}(C)$ implies the existence of an extension $\xi$ as above. Tango, furthermore, gave a lower bound for $\boldsymbol{n}(C)$ :

$$
\frac{g-h(C)}{p-1}-1 \leq \boldsymbol{n}(C),
$$

where $h(C)$ is the rank of the Hasse-Witt matrix of $C$. When $g=h(C)$, this bound is not effective on the positivity of $\boldsymbol{n}(C)$. However, he gave an example of a curve $C$ with positive $\boldsymbol{n}(C)$ and $g=h(C)$ ([loc. cit.]). On the other hand, there exists the following inequality

$$
i \cdot \operatorname{deg} \mathcal{F}-r \cdot \boldsymbol{m}_{i}(\mathcal{F}) \leq i(r-i) g,
$$

where we denote by $\boldsymbol{m}_{i}(\mathcal{F})$ the maximal degree of subsheaves of rank $i$ of a locally free sheaf $\mathcal{F}$ of rank $r$ on $C$. This inequality was proved by Nagata ([5]) for $r=2$, and by Mukai and Sakai ([4]) for $r \geq 3$. Note that the invariant $\boldsymbol{n}(C)$ is just $\boldsymbol{m}_{1}(\mathcal{B})$. By using the inequality (3), we could have a lot of such curves as Tango constructed, as follows.

THEOREM 1.4. In the same notation as above, we have

$$
\frac{g}{p-1}-1 \leq \boldsymbol{n}(C) \leq\left[\frac{2 g-2}{p}\right],
$$

where $[\mathrm{m}]$ denotes the largest integer not greater than $\mathrm{m}$. 
PROOF. By directly applying the inequality (3) to the sheaf $\mathcal{B}$, we have the first inequality. The second inequality is well-known. For reader's convenience, we, however, give a proof. Suppose that $\mathcal{L}$ is an invertible subsheaf of $\mathcal{B}$. Since the sheaf $\mathcal{B}$ is a subsheaf of $F_{*} \Omega_{\tilde{C}}$, we have an injection from $\mathcal{L}$ to $F_{*} \Omega_{C}$. By taking its adjoint map, we have an injection from $\mathcal{L}^{p}$ to $\Omega_{C}$. This implies the second inequality.

Immediately, we have

COROLLARY 1.5. In the same notation as above, we have:

(1) If $g=0$, then $\boldsymbol{n}(C)=-1$;

(2) If $g=p$, then $\boldsymbol{n}(C)=1$;

(3) If $g \geq p$, then $\boldsymbol{n}(C)>0$.

Hence we obtain that any curve with $g \geq p$ has a pre-Tango structure.

COROLlaRY 1.6. For any curve $C$ with $g>n(p-1)$, the sheaf $\mathcal{B}$ contains an invertible subsheaf $\mathcal{L}$ of degree $n$.

2. Forms of the affine line induced from pre-Tango structures. Let $X$ be a scheme over a scheme $S$. We say that $X$ is a form of the affine line over $S$ if there exist a surjective morphism $\bar{S} \rightarrow S$ and an isomorphism over $\bar{S}$ from $X \times{ }_{S} \bar{S}$ to a certain $A^{1}$-bundle over $\bar{S}$. In this section, we shall show that pre-Tango structures over $C$ induce forms of the affine line over $C$ and shall consider geometric properties of completions of such forms, where $C$ is the same as in Section 1. From now on, we suppose the characteristic $p$ is greater than 2. Let $F: \tilde{C} \rightarrow C$ be the relative Frobenius morphism over $k$. To begin with, we state

THEOREM 2.1. Let $n$ be an integer greater than one and relatively prime to $p$. Suppose that we have a pre-Tango structure $\mathcal{L}^{n} \hookrightarrow \mathcal{B}$ on $C$. Then we obtain a scheme $X$ and a smooth morphism $\varphi: X \rightarrow C$ whose base change by $F$ coincides with the geometric line bundle $\tilde{\boldsymbol{L}}=\operatorname{Spec} \operatorname{Symm}\left(F^{*} \mathcal{L}^{-1}\right)$ over $\tilde{C}$. In other words, $\varphi: X \rightarrow C$ is a form of the affine line over $C$.

PROOF. Our proof of this theorem consists of the following three steps:

Step 1. We construct a scheme $Y$ and a morphism $\psi: Y \rightarrow C$ such that every fibre of $\psi$ is a rational curve with one cusp.

Step 2. We construct the required form $X$ by taking the normalization in the function field $k(Y)$ of the geometric line bundle $\boldsymbol{L}^{p}=\operatorname{Spec} \operatorname{Symm}\left(\mathcal{L}^{-p}\right)$ over $C$. Furthermore, we show that $X$ is smooth over $C$.

Step 3. We verify that $X$ is a form of the affine line.

Step 1. Take an affine open covering $\left\{U_{i}\right\}_{i \in I}$ and local generators $\left\{\bar{q}_{i}\right\}_{i \in I}$ of $\mathcal{L}^{n}$ such that each $\bar{q}_{i}$ generates $\mathcal{L}^{n}$ over $U_{i}$. Consider the following extension coming from the inclu$\operatorname{sion} \mathcal{L}^{n} \hookrightarrow \mathcal{B}$ :

$$
0 \rightarrow \mathcal{O}_{C} \rightarrow \mathcal{E} \rightarrow \mathcal{L}^{n} \rightarrow 0
$$


where $\mathcal{E}$ is a locally free $\mathcal{O}_{C}$-module of rank two contained in $F_{*} \mathcal{O}_{\tilde{C}}$. Take an inverse image $q_{i}$ in $\mathcal{E}$ of each $\bar{q}_{i}$. Then $q_{i}$ is a section of $F_{*} \mathcal{O}_{\tilde{C}}$ over $U_{i}$ and $\mathcal{E}$ is generated by 1 and $q_{i}$ over $U_{i}$. Moreover, we can take transition matrices

$$
\left\{\left(\begin{array}{cc}
d_{i j}^{n} & b_{i j} \\
0 & 1
\end{array}\right)\right\}_{i, j \in I}
$$

such that $q_{i}=d_{i j}^{n} q_{j}+b_{i j}$ and that $\left\{d_{i j}\right\}_{i, j \in I}$ are transition functions of $\mathcal{L}$ and $\bar{q}_{i}=d_{i j}^{n} \bar{q}_{j}$ over $U_{i} \cap U_{j}$. Here $d_{i j}^{n}$ and $b_{i j}$ are local sections of $\mathcal{O}_{C}\left(U_{i} \cap U_{j}\right)$. Set $a_{i}=q_{i}^{p}$ for each $i \in I$. Then we know that $a_{i}$ is a section in $\mathcal{O}_{C}\left(U_{i}\right)$ and we have a relation $a_{i}=d_{i j}^{p n} a_{j}+b_{i j}^{p}$ over $U_{i} \cap U_{j}$. For each affine open subset $U_{i}$, consider an affine scheme $Y_{i}=\operatorname{Spec} \mathcal{O}_{C}\left(U_{i}\right)\left[u_{i}, v_{i}\right]$ over $U_{i}$ with relation $v_{i}^{p}=u_{i}^{n}+a_{i}$. Under the relations $v_{i}=d_{i j}^{n} v_{j}+b_{i j}$ and $u_{i}=d_{i j}^{p} u_{j}$, we can then glue $\left\{Y_{i}\right\}_{i \in I}$ together so that we have a scheme $Y$ over $C$. We then know that $Y$ is locally a hypersurface. Let $\psi$ be the structural morphism of $Y$ over $C$, and let $\Sigma$ be the subscheme of $Y$ defined locally by $u_{i}=0$ with the reduced induced structure. From the defining equations of $Y_{i}$ 's, it follows that the restriction of $\psi$ to $Y-\Sigma$ is smooth and that each fibre of $\varphi$ is a rational curve with one cusp of type $v^{p}=u^{n}$.

Step 2. Consider the geometric line bundle $\boldsymbol{L}^{p}=\operatorname{Spec} \operatorname{Symm}\left(\mathcal{L}^{-p}\right)$ over $C$. We can $\left.\operatorname{regard} \boldsymbol{L}^{p}\right|_{U_{i}}=\operatorname{Spec} \mathcal{O}_{C}\left(U_{i}\right)\left[z_{i}\right]$ with $z_{i}=d_{i j}^{-p} z_{j}$. By identifying $z_{i}$ and $u_{i}^{-1}$, we have a purely inseparable extension $k(Y) / k\left(\boldsymbol{L}^{p}\right)$ of degree $p$. Take the normalization $X$ of $\boldsymbol{L}^{p}$ in $k(Y)$. We then have a structural morphism $\varphi: X \rightarrow C$.

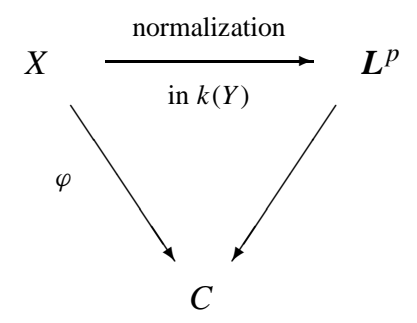

Let us consider local equations defining $X$. Since $v_{i}^{p}=u_{i}^{n}+a_{i}$, we have $v_{i}^{p}=z_{i}^{-n}+a_{i}$ in the function field $k(Y)$. Take the positive integers $r$ and $m$ such that $n+r=m p$ and $r<p$. We can write $z_{i}^{m p} v_{i}^{p}=z_{i}^{r}+a_{i} z_{i}^{m p}$. Set $y_{i}=z_{i}^{m} v_{i}$. We then obtain $y_{i}^{p}=z_{i}^{r}+a_{i} z_{i}^{m p}$. By the Jacobian criterion, we know that the hypersurface which is defined by this equation is smooth over $C$ except for the closed subscheme defined locally by $z_{i}=0$. Therefore, this hypersurface coincides with $X$ outside of that closed subscheme. Since $p$ and $r$ are relatively prime, there exist integers $\alpha$ and $\beta$ such that $\alpha p+\beta r=1$. Set $x_{i}=y_{i}^{\beta} z_{i}^{\alpha}$ for each $i \in I$. We then have $x^{p}=z_{i}\left(1+a_{i} z_{i}^{n}\right)^{\beta}$ and $x_{i}^{r}=y_{i}\left(1+a_{i} z_{i}^{n}\right)^{-\alpha}$. Near $z_{i}=0$, the hypersurface defined by $x^{p}=z_{i}\left(1+a_{i} z_{i}^{n}\right)^{\beta}$ is smooth and coincides with $X$. Moreover, it follows that $\varphi: X \rightarrow C$ is smooth and that $\mathcal{O}_{X}\left(\varphi^{-1}\left(U_{i}\right)\right)$ contains $\mathcal{O}_{C}\left(U_{i}\right)\left[y_{i}, z_{i}\right]$.

Step 3. Consider the geometric line bundle $\boldsymbol{L}=\operatorname{Spec} \operatorname{Symm}\left(\mathcal{L}^{-1}\right)$ over $C$. We know that $\boldsymbol{L}$ is defined by the transition functions $\left\{d_{i j}\right\}_{i, j \in I}$ and that $\tilde{\boldsymbol{L}}$ is the pull-back of $\boldsymbol{L}$. Recall the relation $y_{i}^{p}=z_{i}^{r}+a_{i} z_{i}^{m p}$ with $a_{i} \in k(C)$. Let $\alpha$ and $\beta$ be the same integers as in Step 2, 
and set $s_{i}=\left(y_{i}-q_{i} z_{i}^{m}\right)^{\beta} z_{i}^{\alpha}$. Then we have $s_{i}^{p}=z_{i}$ and $s_{i}^{r}=y_{i}-q_{i} z_{i}^{m}$. Since $z_{i}=d_{i j}^{-p} z_{j}$, we know $s_{i}=d_{i j}^{-1} s_{j}$. So, we can regard $\left.\boldsymbol{L}\right|_{U_{i}}=\operatorname{Spec} \mathcal{O}_{C}\left(U_{i}\right)\left[s_{i}\right]$ and $\left.\tilde{\boldsymbol{L}}\right|_{\tilde{U}_{i}}=\operatorname{Spec} \mathcal{O}_{\tilde{C}}\left(\tilde{U}_{i}\right)\left[s_{i}\right]$, where $\tilde{U}_{i}$ is the inverse image of $U_{i}$ by $F$. In order to show that the base change $\tilde{\varphi}: \tilde{X} \rightarrow \tilde{C}$ of $\varphi: X \rightarrow C$ coincides with $\tilde{\boldsymbol{L}}$ over $\tilde{C}$, we consider the coordinate ring $\mathcal{O}_{\tilde{X}}\left(\tilde{\varphi}^{-1}\left(\tilde{U}_{i}\right)\right)$. By virtue of Step 2, we have an inclusion $\mathcal{O}_{\tilde{C}}\left(\tilde{U}_{i}\right)\left[s_{i}^{p}, s_{i}^{r}\right] \subset \mathcal{O}_{\tilde{X}}\left(\tilde{\varphi}^{-1}\left(\tilde{U}_{i}\right)\right)$. Since $X$ is smooth, we know that the coordinate $\operatorname{ring} \mathcal{O}_{\tilde{X}}\left(\tilde{\varphi}^{-1}\left(\tilde{U}_{i}\right)\right)$ is normal. Therefore, it follows that $\mathcal{O}_{\tilde{X}}\left(\tilde{\varphi}^{-1}\left(\tilde{U}_{i}\right)\right)$ is the normalization of $\mathcal{O}_{\tilde{C}}\left(\tilde{U}_{i}\right)\left[s_{i}^{p}, s_{i}^{r}\right]$, which coinsides with $\mathcal{O}_{\tilde{C}}\left(\tilde{U}_{i}\right)\left[s_{i}\right]$.

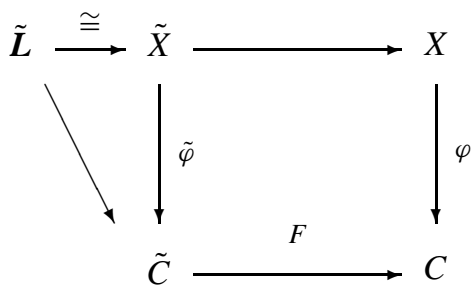

Hence we obtain an isomorphism from $\tilde{X}$ to $\tilde{\boldsymbol{L}}$ over $\tilde{C}$. This completes the proof of the theorem.

REMARK 2.2. From the argument above, it follows that we obtain a form of the affine line over $C$ if there exists an inclusion $\mathcal{L}^{n} \hookrightarrow \mathcal{B}$, where $n$ is an integer greater than one and relatively prime to $p$ and where $\mathcal{L}$ is an invetible sheaf not necessarily positive.

Next, we shall consider a completion of the form obtained in the previous theorem. We retain the same notation and assumptions as in the previous theorem and proof. Consider the ruled surface $\boldsymbol{P}\left(\mathcal{L}^{p} \oplus \mathcal{O}\right) \rightarrow C$ and take the normalization $Z$ in $k(X)$, i.e., in $k(Y)$. We then have two open immersions $X \rightarrow Z$ and $Y \rightarrow Z$. Let $\Phi: Z \rightarrow C$ be the structural morphism. By considering the local equations of $X$ and of $Y$, we know that $\Phi$ is a fibration such that each fibre is a rational curve with one cusp of type $v^{p}=u^{n}$. Recall the subscheme $\Sigma$ defined by the ideal sheaf generated locally by $u_{i}$ (cf. Step 1 in the previous proof). From the local equations of $Y$, it follows that $\Sigma$ is the moving cuspidal singularities of the fibration $\Phi$.

REMARK 2.3 (cf. [6], [7]). By applying the Jacobian criterion, we know that the completions of the forms mentioned above have no singular points when they are induced from Tango structures. Some of them are well-known. Indeed, Raynaud constructed a nonsingular surface by taking the double covering of the ruled surface $\boldsymbol{P}(\mathcal{E})$, where $\mathcal{E}$ is the same extension as (4) in Step 1 of the previous proof with a Tango structure $\mathcal{L}^{2} \hookrightarrow \mathcal{B}$. That surface coincides with one of the completions mentioned above. Moreover, he showed that, on that surface, there exists an ample invertible sheaf which gives a counter-example to the Kodaira vanishing theorem in positive characteristic. The ample invertible sheaf coincides with $\mathcal{O}_{Z}(E) \otimes \Phi^{*}(\mathcal{L})$ in our notation, where $E$ is a section of $\Phi$ determined locally by $x_{i}=0$, i.e., $E=Z-Y$. 
Meanwhile, by taking the quotients of ruled surfaces by $p$-closed rational vector fields, Russell generally constructed a certain class of uni-ruled surfaces, which contains the completions mentioned above. Furthermore, he described specific examples, which are smooth surfaces of general type with non-trivial global vector fields. Those examples coincide with the completions of the forms which are induced from Tango structures of the form $\mathcal{L}^{n} \hookrightarrow \mathcal{B}$ with $n \equiv-1(\bmod p)$ in our notation.

Since the above-mentioned famous examples are induced from Tango structures, we can easily verify that their Euler numbers are negative and that they are not ruled surfaces. Hence we know that those examples cannot be lifted to characteristic zero. On the other hand, the completions of the forms induced from pre-Tango structures, discussed in the present article, have some complicated singular points. So, in general, it is hard to compute the numerical invariant of their desingularization. We, however, know the local equations defining $Z$. Therefore, we can treat the differential forms intelligibly. Indeed, we have the following theorem:

THEOREM 2.4 (cf. [9]). Retain the same notation and assumptions as above. Suppose that we have a pre-Tango structure $\mathcal{L}^{n} \hookrightarrow \mathcal{B}$ on $C$ such that $n>p^{2}$. Then $Z$ has non-closed global differential 1-forms if $H^{0}\left(C, \mathcal{L}^{p}\right) \underset{f}{\subsetneq} H^{0}\left(C, \mathcal{L}^{p} \otimes_{\mathcal{O}_{C}} \Phi_{*} \mathcal{O}_{\Sigma}\right)$.

Proof. Since $Y$ is defined locally by $v_{i}^{p}=u_{i}^{n}+a_{i}$, we have $-n u_{i}^{n-1} \mathrm{~d} u_{i}=\mathrm{d} a_{i}$ by exterior differentiation. Let $\mathcal{P}$ be the $\mathcal{O}_{C}$-submodule of $\Omega_{C / k}$ locally generated by $\mathrm{d} a_{i}$, where $\Omega_{C / k}$ is the sheaf of differentials on $C$. We then obtain an injection $\Phi^{*} \mathcal{P} \otimes_{\mathcal{O}_{Z}} \mathcal{O}_{Z}((n-$ 1) $\Sigma) \rightarrow \Omega_{Z / k}^{1}$, where $\Omega_{Z / k}^{1}$ is the sheaf of differentials on $Z$. We consider the global differential 1-forms in $\Phi^{*} \mathcal{P} \otimes \mathcal{O}_{Z} \mathcal{O}_{Z}((n-1) \Sigma)$. Over $U_{i}$, we have

$$
\begin{aligned}
\mathrm{d} u_{i} & =-\frac{1}{n} z_{i}^{n-1} \mathrm{~d} a_{i}, \\
v_{i} \mathrm{~d} u_{i} & =-\frac{1}{n} y_{i} z_{i}^{m(p-1)-r-1} \mathrm{~d} a_{i}, \\
& \vdots \\
v_{i}^{l} \mathrm{~d} u_{i} & =-\frac{1}{n} y_{i}^{l} z_{i}^{m(p-l)-r-1} \mathrm{~d} a_{i} .
\end{aligned}
$$

The left hand side of each equation says that the differential form is regular on $Y$ and the right hand side says that the differential form is regular on $X$ when $m(p-l)-r-1>0$. Since $n>p^{2}$, we have $m>r+1$. Therefore, we obtain that $\mathrm{d} u_{i}, v_{i} \mathrm{~d} u_{i}, \ldots, v_{i}^{p-1} \mathrm{~d} u_{i}$ are sections in $\Phi^{*} \mathcal{P} \otimes \mathcal{O}_{Z}((n-1) \Sigma)\left(\Phi^{-1}\left(U_{i}\right)\right)$ and so in $\mathcal{P} \otimes \Phi_{*} \mathcal{O}_{Z}((n-1) \Sigma)\left(U_{i}\right)$. Let $\mathcal{Q}$ be the $\mathcal{O}_{C}$-submodule of $\mathcal{P} \otimes_{\mathcal{O}_{C}} \Phi_{*} \mathcal{O}_{Z}((n-1) \Sigma)$ generated locally by $\mathrm{d} u_{i}$. Then $\mathcal{Q}$ is isomorphic to $\mathcal{L}^{p}$. Consider the direct image of the structure sheaf $\mathcal{O}_{\Sigma}$ of $\Sigma$ by $\Phi$. Since $\Phi_{*} \mathcal{O}_{\Sigma}$ is generated locally by $1, v_{i}, \ldots, v_{i}^{p-1}$ as an $\mathcal{O}_{C}$-module, we have an inclusion $\mathcal{Q} \otimes_{\mathcal{O}_{C}} \Phi_{*} \mathcal{O}_{\Sigma} \subset$ $\mathcal{P} \otimes \mathcal{O}_{C} \Phi_{*} \mathcal{O}_{Z}((n-1) \Sigma)$. We summarize the above-mentioned inclusions of $\mathcal{O}_{C}$-modules as follows:

$$
\mathcal{Q} \subset \mathcal{Q} \otimes_{\mathcal{O}_{C}} \Phi_{*} \mathcal{O}_{\Sigma} \subset \mathcal{P} \otimes_{\mathcal{O}_{C}} \Phi_{*} \mathcal{O}_{Z}((n-1) \Sigma) \subset \Phi_{*} \Omega_{Z / k}^{1}
$$


Since $\mathcal{Q}$ is locally generated by $\mathrm{d} u_{i}$, any section of $\mathcal{Q}$ is a closed differential 1 -form on $Z$. Meanwhile, $\mathcal{Q} \otimes_{\mathcal{O}_{C}} \Phi_{*} \mathcal{O}_{\Sigma}$ is locally generated by $\mathrm{d} u_{i}, v_{i} \mathrm{~d} u_{i}, \ldots, v_{i}^{p-1} \mathrm{~d} u_{i}$. So we conclude that the sections of $\mathcal{Q} \otimes_{\mathcal{O}_{C}} \Phi_{*} \mathcal{O}_{\Sigma}$ which are not in $\mathcal{Q}$ are non-closed differential 1-forms on $Z$. Hence we arrive at the required assertion.

REMARK 2.5. Let $\sigma: Z^{*} \rightarrow Z$ be a desingularization. Since there is a natural injection $\sigma^{*} \Omega_{Z / k} \rightarrow \Omega_{Z^{*} / k}$, we know that, if $Z$ has non-closed global differential 1-forms, then so does $Z^{*}$. In this case, the Hodge to de Rham spectral sequence of $Z^{*}$ does not degenerate in $E_{1}$. Hence, by Corollaire 2.4 of [1], we know that $Z^{*}$ can not be lifted over the ring of Witt-vectors of length two.

REMARK 2.6. If $\mathcal{L}^{n} \hookrightarrow \mathcal{B}$ is a Tango structure, then we have $H^{0}\left(Z, \Phi^{*} \mathcal{P} \otimes \mathcal{O}_{Z}\right.$ $\left.\mathcal{O}_{Z}((n-1) \Sigma)\right)=H^{0}\left(Z, \Omega_{Z / k}^{1}\right)$ (cf. Takeda [8]). However, the authors do not know whether the same equation holds in general. That is obstructed by the existence of the singular points. Meanwhile, the argument in the previous proof treats only the sections of the form $v_{i}^{l} \mathrm{~d} u_{i}$ in $\Phi^{*} \mathcal{P} \otimes \mathcal{O}_{Z} \mathcal{O}_{Z}((n-1) \Sigma)$. So, by our method, it is hard to investigate all differential 1-forms on $Z^{*}$ and to state a necessary and sufficient conditon for the existence of non-closed global differential 1-forms on $Z^{*}$. There seems to be a need of another method.

REMARK 2.7. Let $\hat{C}$ be the curve obtained by gluing $\left\{\operatorname{Spec} \mathcal{O}_{C}\left(U_{i}\right)\left[q_{i}\right]\right\}_{i \in I}$ together and $\hat{F}: \hat{C} \rightarrow C$ the canonical morphism, where $q_{i}$ 's are the same as in Step 1 of the proof of Theorem 2.1. Clearly, the normalization of $\hat{C}$ is none other than $\tilde{C}$ and they coincide provided that $\mathcal{L}^{n} \hookrightarrow \mathcal{B}$ is a Tango structure. Moreover, by identifying $q_{i}$ and $v_{i}$ on $\Sigma$, we know that there exists a closed immersion $\hat{C} \rightarrow Z$ whose image coincides with $\Sigma$.

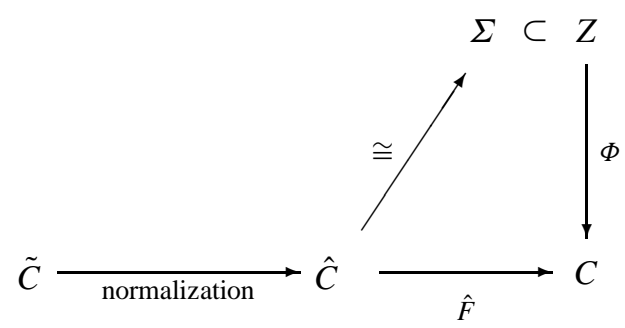

Hence, in the same notation and under the same assumptions as in the previous theorem, if $H^{0}\left(C, \mathcal{L}^{p}\right) \varsubsetneqq H^{0}\left(C, \mathcal{L}^{p} \otimes \mathcal{O}_{C} \hat{F}_{*} \mathcal{O}_{\hat{C}}\right)$, then we have that $Z$ has non-closed global differential 1 -forms.

3. Pre-Tango structures on hyperelliptic curves. We have already seen that any curve of sufficiently high genus has pre-Tango structures (cf. Section 1). In this section, by using the Cartier operator, we shall explicitly describe some pre-Tango structures on hyperelliptic curves and shall consider them more precisely. Let $C$ be a hyperelliptic curve of genus $g$ over $k$ of characteristic $p>2$. From now on, we employ the absolute Frobenius morphism $F: C \rightarrow C$ instead of the relative Frobenius $k$-morphism. Then the sheaf $\mathcal{B}$ is defined to be $F_{*} \mathcal{O}_{C} / \mathcal{O}_{C}$. We use the Cartier operator $\mathcal{C}: F_{*} \Omega_{C} \rightarrow \Omega_{C}$, which is defined as follows. Let $t$ 
be a local parameter at a point on $C$. Then any rational function $f$ of $C$ can be written as

$$
f=f_{0}^{p}+f_{1}^{p} t+\cdots+f_{p-1}^{p} t^{p-1}
$$

with $f_{i} \in k(C)$. For a differential 1-form $f \mathrm{~d} t$ on $C$, we set

$$
\mathcal{C}(f \mathrm{~d} t)=f_{p-1} \mathrm{~d} t
$$

We can verify that the Cartier operator $\mathcal{C}$ does not depend on the choice of a local parameter $t$. Moreover, $\mathcal{C}$ induces a homomorphism $\mathcal{C}: F_{*} \Omega_{C} \rightarrow \Omega_{C}$ of sheaves which induces a short exact sequence

$$
0 \longrightarrow \mathcal{B} \longrightarrow F_{*} \Omega_{C} \stackrel{\mathcal{C}}{\longrightarrow} \Omega_{C} \longrightarrow 0 .
$$

The following lemma states the existence of a pre-Tango structure of high degree.

Lemma 3.1. Let $C$ be a hyperelliptic curve defined by an equation $y^{2}=f(x)$ such that $\operatorname{deg} f=2 g+1$ and $f(0)=0$. Suppose that there exists an integer $i$ with $0 \leq i \leq g-1$ such that $\mathcal{C}\left(x^{i} \mathrm{~d} x / y\right)=0$. If $n$ is a natural number relatively prime to $p$ with $2 g-2-2 i-2 p<$ $n p \leq 2 g-2-2 i$, then there exists a pre-Tango structure of degree $n$ on $C$.

Proof. By the assumption, we know that the hyperelliptic curve $C$ has a double covering $\pi: C \rightarrow \boldsymbol{P}^{1} ;(x, y) \mapsto x$ such that two Weierstrass points $P_{0}$ and $P_{\infty}$ are lying over $x=0$ and $x=\infty$, respectively. Then the divisor $\left(x^{i} \mathrm{~d} x / y\right)$ of the differential form $x^{i} \mathrm{~d} x / y$ is $2 i P_{0}+(2 g-2-2 i) P_{\infty}$. Take a natural number $n$ relatively prime to $p$ such that $2 g-2-2 i-2 p<n p \leq 2 g-2-2 i$. Then the divisor $\left(x^{i} \mathrm{~d} x / y\right)-n p P_{\infty}$ is effective. Hence we have an inclusion $\mathcal{O}_{C}\left(n p P_{\infty}\right) \subset \Omega_{C}$ and so we have its adjoint $\mathcal{O}_{C}\left(n P_{\infty}\right) \subset F_{*} \Omega_{C}$. Meanwhile, by the short exact sequenc (5), we have that the section $x^{i} \mathrm{~d} x / y$ comes from $\mathcal{B}$ since $\mathcal{C}\left(x^{i} \mathrm{~d} x / y\right)=0$. Hence we get an inclusion $\mathcal{O}_{C}\left(n P_{\infty}\right) \subset \mathcal{B}$. So $\mathcal{O}_{C}\left(n P_{\infty}\right)$ is a pre-Tango structure.

Let us consider a pre-Tango structure $\mathcal{L}^{n} \hookrightarrow \mathcal{B}$ satisfying:

The degree $n$ is relatively prime to $p$ and greater than $p^{2}$, and

$$
H^{0}\left(C, \mathcal{L}^{p}\right) \varsubsetneqq H^{0}\left(C, \mathcal{L}^{p} \otimes_{\mathcal{O}_{C}} \hat{F}_{*} \mathcal{O}_{\hat{C}}\right) .
$$

Here $\hat{C}, \hat{F}$ are the same as in Remark 2.7, i.e., $\hat{C}$ is obtained by gluing $\left\{\operatorname{Spec} \mathcal{O}_{C}\left(U_{i}\right)\left[q_{i}\right]\right\}_{i \in I}$, where $q_{i}$ 's are local generators of $\mathcal{E}$, subjected to the relation $q_{i}=d_{i j}^{n} q_{j}+b_{i j}$ and $\hat{F}: \hat{C} \rightarrow C$ is the canonical morphism. Remarks 2.5 and 2.7 say that we can construct a smooth complete surface which has non-closed global differential 1 -forms by using a pre-Tango structure $\mathcal{L}^{n}$ satisfying $(*)$. Let us consider curves, especially hyperelliptic curves, having such pre-Tango structures. More precisely, let $\mathcal{M}_{g}$ be the moduli space of curves of genus $g$ and consider the following locus:

$$
\mathcal{T}_{g}=\left\{C \in \mathcal{M}_{g} \mid C \text { has a pre-Tango structure } \mathcal{L}^{n} \text { satisfying }(*)\right\} .
$$

To close this article, we state the following theorem: 
THEOREM 3.2. If $2 g-4 p \geq p^{3}$ and $p \geq 11$, then the locus $\mathcal{T}_{g}$ contains a variety of dimension $g-1$.

Proof. Consider the subvariety $\mathcal{H}_{g}$ of $\mathcal{M}_{g}$ consisting of hyperelliptic curves of genus $g$. It is well-known that the dimension of $\mathcal{H}_{g}$ is $2 g-1$. Since each curve in $\mathcal{H}_{g}$ is given by an equation of type $y^{2}=x(x-1)\left(x-a_{1}\right) \cdots\left(x-a_{2 g-1}\right)$, we have a rational mapping $A^{2 g-1} \rightarrow \mathcal{H}_{g}$ whose image coinsides with $\mathcal{H}_{g}$. Let $C$ be a curve in $\mathcal{H}_{g}$ defined by the equation $y^{2}=x(x-1)\left(x-a_{1}\right) \cdots\left(x-a_{2 g-1}\right)$, and let

$$
\left(\begin{array}{ccc}
c_{11} & \ldots & c_{1 g} \\
\vdots & & \vdots \\
c_{g 1} & \ldots & c_{g g}
\end{array}\right)
$$

be the Hasse-Witt matrix of $C$, i.e., the matrix representing the mapping on $H^{0}\left(C, \Omega_{C}\right)$ induced from the Cartier operator $\mathcal{C}$, with respect to the basis $\left\{\mathrm{d} x / y, x \mathrm{~d} x / y, \ldots, x^{g-1} \mathrm{~d} x / y\right\}$. Note that all entries of this matrix are polynomials in $a_{1}, \ldots, a_{2 g-1}$ (see Yui [12] for example, where the Hasse-Witt matrix in our sense is called the Cartier-Manin matrix and the Cartier operator is called the modified Cartier operator). For $1 \leq i \leq g$, consider the subvariety $S_{i}$ of $A^{2 g-1}$ defined by $c_{i 1}=\cdots=c_{i g}=0$. Namely, $S_{i}$ is the subvariety consisting of the points corresponding to curves such that all entries in the $i$-th columns of their Hasse-Witt matrices are zero. Let $V_{i}$ be the image in $\mathcal{H}_{g}$ of $S_{i}$. If $V_{i}$ is not empty, then we have $\operatorname{dim} V_{i} \geq g-1$.

Suppose that $V_{i}$ is not empty with $i \leq p$. Let $C$ be a hyperelliptic curve corresponding to a point in $V_{i}$. Then we have $\mathcal{C}\left(x^{i-1} \mathrm{~d} x / y\right)=0$. Since $i \leq p$ and $2 g-4 p \geq p^{3}$, we have $2 g-2-2(i-1)-2 p \geq p^{3}$. By virtue of the previous lemma, we have a pre-Tango structure $\mathcal{L}^{n}$ with $\mathcal{L}=\mathcal{O}_{C}\left(P_{\infty}\right)$ and $n>p^{2}$. Consider the exact sequence

$$
0 \rightarrow \mathcal{O}_{\hat{C}} \rightarrow \mathcal{O}_{\tilde{C}} \rightarrow \mathcal{O}_{\tilde{C}} / \mathcal{O}_{\hat{C}} \rightarrow 0
$$

on $C$, where $\tilde{C}$ is the normalization of $\hat{C}$ (cf. Remark 2.7). Since $n p>2 g-2-2(i-1)-2 p$, we know that $\mathcal{O}_{\tilde{C}} / \mathcal{O}_{\hat{C}}$ is a torsion sheaf of degree less than $2(i-1)+2 p$. By tensoring $\mathcal{L}^{p}$ over $\mathcal{O}_{C}$ to (6), we have

$$
0 \rightarrow \mathcal{O}_{\hat{C}} \otimes \mathcal{L}^{p} \rightarrow \mathcal{O}_{\tilde{C}} \otimes \mathcal{L}^{p} \rightarrow \mathcal{O}_{\tilde{C}} / \mathcal{O}_{\hat{C}} \rightarrow 0
$$

Since $\mathcal{L}=\mathcal{O}_{C}\left(P_{\infty}\right)$, we obtain that $\operatorname{dim} H^{0}\left(C, \mathcal{L}^{p}\right)=(p-1) / 2+1$. Moreover, we have that $\mathcal{O}_{\tilde{C}} \otimes \mathcal{L}^{p}$ is isomorphic to $\mathcal{L}^{p^{2}}$. Therefore, we have that $\operatorname{dim} H^{0}\left(\mathcal{O}_{\tilde{C}} \otimes \mathcal{L}^{p}\right)=\left(p^{2}-1\right) / 2+1$. Since $\operatorname{deg} \mathcal{O}_{\tilde{C}} / \mathcal{O}_{\hat{C}}<2 i-2+2 p \leq 4 p-2$, we know that $\operatorname{dim} H^{0}\left(\mathcal{O}_{\hat{C}} \otimes \mathcal{L}^{p}\right)>\left(p^{2}-1\right) / 2+$ $1-4 p+2$. Hence $p \geq 11$ implies that $H^{0}\left(C, \mathcal{L}^{p}\right) \varsubsetneqq H^{0}\left(C, \mathcal{L}^{p} \otimes_{\mathcal{O}_{C}} \hat{F}_{*} \mathcal{O}_{\hat{C}}\right)$. Therefore, we conclude that $V_{i}$ is contained in $\mathcal{T}_{g}$. Now we have only to show that one of $\left\{V_{i} \mid i \leq p\right\}$ is not empty.

Set $d=2 g+1$ and let $C$ be the hyperelliptic curve determined by $y^{2}=x^{d}-x$. Consider the differential form $\mathrm{d} x / y$ and its image by the Cartier operator. We have

$$
y \mathcal{C}\left(\frac{\mathrm{d} x}{y}\right)=\mathcal{C}\left(y^{p-1} \mathrm{~d} x\right)=\mathcal{C}\left(\left(x^{d}-x\right)^{(p-1) / 2} \mathrm{~d} x\right) .
$$


Only the terms

$$
x^{(p-1) / 2}, \quad x^{d+(p-1) / 2-1}, \quad \ldots, \quad x^{d((p-1) / 2-1)+1}, \quad x^{d(p-1) / 2}
$$

appear in $\left(x^{d}-x\right)^{(p-1) / 2}$. If $\mathcal{C}(\mathrm{d} x / y)=0$, then we have that $V_{1}$ is not empty. Assume that $\mathcal{C}(\mathrm{d} x / y) \neq 0$. Then there exists an integer $l$ such that $0 \leq l \leq(p-1) / 2$ and $d l+(p-1) / 2-$ $l \equiv p-1$ i.e., $l(d-1)+(p-1) / 2 \equiv p-1(\bmod p)$. Note that $d-1 \not \equiv 0(\bmod p)$. Let $j$ be the integer such that $0 \leq j \leq p-1$ and $j \equiv(l+1)(d-1)(\bmod p)$. Consider the differential form $x^{j} \mathrm{~d} x / y$. We then have $j+(p-1) 2 \equiv(l+1)(d-1)+(p-1) / 2$. Moreover, since the mapping $\boldsymbol{Z} / p \boldsymbol{Z} \rightarrow \boldsymbol{Z} / p \boldsymbol{Z} ; l \mapsto l(d-1)+(p-1) / 2$ is bijective, we know that the integers $j+(p-1) / 2, j+d+(p-1) / 2-1, \ldots, j+d((p-1) / 2-1)+1, j+d(p-1) / 2$ are not equivalent to $p-1$ modulo $p$. So, we have $\mathcal{C}\left(x^{j} \mathrm{~d} x / y\right)=0$. Hence we obtain that $V_{j+1}$ is not empty.

\section{REFERENCES}

[ 1 ] P. Deligne And L. Illusie, Relèvements modulo $p^{2}$ et décomposition du complex de de Rham, Invent. Math. 89 (1987), 247-270.

[ 2 ] A. Hirschowitz, Problèmes de Brill-Noether en rang supérieur, C. R. Acad. Sci. Paris Sér. I Math. 307 (1988), 153-156.

[ 3 ] W. E. LANG, Quasi-elliptic surfaces in characteristic three, Ann. Sci. Ecole Norm. Sup. (4) 12 (1979), 473500 .

[ 4 ] S. Mukai AND F. SAKaI, Maximal subbundles of vector bundles on a curve, Manuscripta Math. 52 (1985), 251-256.

[ 5 ] M. NAGATA, On self-intersection number of a section on a ruled surface, Nagoya Math. J. 37 (1970), 191-196.

[ 6 ] M. RaYnaUd, Contre-exemple au "Vanishing Theorem" en caractéristique $p>0$, C. P. Ramanujan-A Tribute, pp. 273-278, Tata Inst. Fund. Res. Studies in Math. 8, Springer-Verlag, Berlin, Heidelberg, New York, 1978.

[ 7 ] P. RUSSELL, Factoring the Frobenius morphism of an algebraic surface, Algebraic Geometry, Bucharest 1982 (Bucharest, 1982), 366-380, Lecture Notes in Math. 1056, Springer-Verlag, Berlin, Heidelberg, New York, Tokyo, 1984.

[ 8 ] Y. TAKEDA, Vector fields and differential forms on generalized Raynaud surfaces, Tôhoku Math. J. 44, (1992) 359-364.

[ 9 ] Y. TAKEDA, Groups of Russell type over a curve, J. Pure and Applied Algebra 128 (1998), 93-108; Corrigendum 148 (2000), 317-318.

[10] H. TANGO, On the behavior of extensions of vector bundles under the Frobenius map, Nagoya Math. J. 48 (1972), 73-89.

[11] K. YOKOGAWA, On maximal subbundles of vector bundles on a curve, Master's Thesis, unpublished.

[12] N. YUI, On the Jacobian varieties of hyperelliptic curves over fields of characteristic $p>2$, J. Algebra 52 (1978), 378-410.

DEPARTMENT OF MATHEMATICS NARA WOMEN'S UNIVERSITY

NARA 630-8506

JAPAN
DEPARTMENT OF MATHEMATICS

OCHANOMIZU UNIVERSITY

OTSUKA 2-1-1, BUNKYO-KU

TOKYO 112-0012

JAPAN 